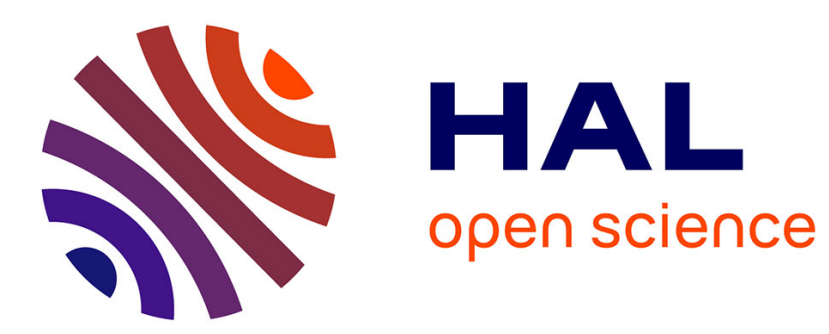

\title{
The ethics of innovation for Alzheimer's disease: the risk of overstating evidence for metabolic enhancement protocols
}

Timothy Daly, Ignacio Mastroleo, David Gorski, Stéphane Epelbaum

\section{- To cite this version:}

Timothy Daly, Ignacio Mastroleo, David Gorski, Stéphane Epelbaum. The ethics of innovation for Alzheimer's disease: the risk of overstating evidence for metabolic enhancement protocols. Theoretical Medicine and Bioethics, 2021, 10.1007/s11017-020-09536-7 . hal-03114575

\section{HAL Id: hal-03114575 \\ https://hal.science/hal-03114575}

Submitted on 19 Jan 2021

HAL is a multi-disciplinary open access archive for the deposit and dissemination of scientific research documents, whether they are published or not. The documents may come from teaching and research institutions in France or abroad, or from public or private research centers.
L'archive ouverte pluridisciplinaire HAL, est destinée au dépôt et à la diffusion de documents scientifiques de niveau recherche, publiés ou non, émanant des établissements d'enseignement et de recherche français ou étrangers, des laboratoires publics ou privés. 


\title{
The ethics of innovation for Alzheimer's disease: the risk of overstating evidence for metabolic enhancement protocols
}

\author{
Timothy Daly, ${ }^{*}$ Ignacio Mastroleo, ${ }^{\dagger}$ David Gorski, ${ }^{\ddagger}$ Stéphane Epelbaum ${ }^{\S}$ \\ timothy.daly@paris-sorbonne.fr
}

\begin{abstract}
Medical practice is ideally based on robust, relevant research. However, the lack of diseasemodifying treatments for Alzheimer's disease has motivated "innovative practice" to improve patients' well-being despite insufficient evidence for the regular use of such interventions in health systems treating millions of patients. Innovative or new non-validated practice poses at least three distinct ethical questions: first, about the responsible application of new non-validated practice to individual patients (clinical ethics); second, about the way in which data from new non-validated practice are communicated via the scientific and lay press (scientific communication ethics); and third, about the prospect of making new non-validated interventions widely available before more definitive testing (public health ethics). We argue that the authors of metabolic enhancement protocols for Alzheimer's disease have overstated the evidence in favor of these interventions within the scientific and lay press, failing to communicate weaknesses in their data and uncertainty about their conclusions. Such unmeasured language may create false hope, cause financial harm, undermine informed consent, and frustrate the production of generalizable knowledge necessary to face the societal problems posed by this devastating disease. We therefore offer more stringent guidelines for responsible innovation in the treatment of Alzheimer's disease.
\end{abstract}

Keywords Innovation; Alzheimer's disease; Dementia; Diffusion of innovation; Integrative medicine; Clinical ethics; Biomedical ethics

\section{Introduction}

Research and practice in medicine are distinguished by their intention $[1,2]$ : the primary aim of research is to contribute to "generalizable knowledge," while the primary aim of practice is to

\footnotetext{
${ }^{*}$ Sorbonne University, Paris, France | email: timothy.daly@paris-sorbonne.fr | ORCID: 0000-0003-1650-242X

† National Scientific and Technical Research Council, Buenos Aires, Argentina | email: ignaciomastro@gmail.com | ORCID: 0000-0002-8243-9943

^ Barbara Ann Karmanos Cancer Institute, Detroit, MI, USA | email: gorskid@med.wayne.edu | ORCID: 00000002-9805-6699

$\S$ Institut du Cerveau et de la Moelle épinière (Brain and Spine Institute), Paris, France | email: stephane.epelbaum@aphp.fr | ORCID: 0000-0003-4059-2891
} 
"enhance the well-being of a particular individual or groups of individuals" [2, p. 3]. By

providing medical practice with robust treatment data, biomedical research has reduced the burden of public health scourges such as cancer and HIV/AIDS. Yet despite decades of investment in and clinical trials of disease-modifying agents, research has so far not achieved disease-modifying therapeutic results for Alzheimer's disease (AD), the most common cause of the 50 million cases of dementia worldwide and a major source of disease burden. There is understandably huge demand for research solutions to AD, raising a number of ethical issues which result from long clinical trials with cognitively-declining AD patients [3].

Progress made in AD research has diminished centuries of prejudice and conceptual confusion surrounding so-called "senile dementia," and has separated AD from other contributing causes of dementia, allowing for earlier diagnosis and targeted treatments in AD patients [4]. Symptomatic AD patients are, however, a very treatment-resist population. Since it is well recognized that initiation of the disease process precedes symptom presentation by decades, scientists are pleading for earlier pharmacological interventions and for theories and treatments which take into account both genetic and lifestyle contributions to AD outcomes [5, $6]$.

Diagnosis of $\mathrm{AD}$ is currently confirmed via clinico-biological examination. Standard care practices include the prescription of pharmaceutical (acetylcholinesterase inhibitors or memantine) and non-pharmaceutical therapies (speech therapy, psychotherapy, mindfulness, physical exercise, art therapy, etc.). Comorbidities, such as depression or vascular disease, are also identified and treated. Finally, medico-social actions are undertaken as needed, and the patient may be included in clinical trials to test disease-modifying agents. Existing "anti- 
dementia" drugs do not halt the disease; thus, AD-related cognitive decline is understood as currently irreversible.

Given the present lack of curative and preventative therapies, some clinicians engage in socalled "innovative practice" for AD patients without robust supporting evidence. Such interventions are ethically permissible if they address an unmet serious or life-threatening medical need and comply with ethical principles $[7,8]$.

The term "innovation" in medicine is confusing, since it can encompass both research and practice — discrete activities with different principles governing their ethical evaluation and harm-benefit analysis $[9,10,11]$. Moreover, an important distinction is made within health care between validated and non-validated practice, based on whether there is sufficient evidence of the safety or efficacy of a class of interventions to justify their regular use in the health care of millions of patients $[11,12]$. To avoid this confusion, we follow the Belmont Report and others in identifying innovation with non-validated practice $[1,2,11]$, a class of diagnostic, preventive, or therapeutic interventions, primarily to benefit patients, for which there is insufficient evidence of safety or efficacy to warrant regular use in medical practice. More specifically, what we term innovative practice refers to neither research nor validated practice, but to initial or recent usage of non-validated interventions with the aim of benefiting individual patients—or "new nonvalidated practice" (NNVP) [12]. While this definition is ethically neutral, there are at least three interrelated ethical dimensions along which to evaluate responsible NNVP.

First is the dimension of clinical ethics, which concerns the ethical utilization of innovative therapeutics and technologies to treat individual patients. Per paragraph 37 of the Declaration of Helsinki, the unmet medical needs of AD patients present an opportunity for responsible NNVP 
use: "In the treatment of an individual patient, where proven interventions ... have been ineffective, the physician, after seeking expert advice, with informed consent from the patient or a legally authorised representative, may use an unproven intervention if in the physician's judgement it offers hope of saving life, re-establishing health or alleviating suffering" [8].

Second is the dimension of scientific communication ethics, which involves the ethics of reporting NNVP data in the scientific and lay literature. Ethical frameworks stipulate that clinician-scientists should use their experience with innovative practice to contribute to generalizable research [7]. According to Helsinki paragraph 36, "Researchers ... and publishers all have ethical obligations with regard to the publication and dissemination of the results of research. Researchers ... are accountable for the completeness and accuracy of their reports" [8]. Since the pathway from evidence generation to consumption contains many steps which can lead to misinformation [13], publishing the results of NNVP may generate undesirable consequences, accelerating the adoption of unvalidated practices by clinicians_-known as "runaway diffusion" [9] - despite insufficient data to establish their safety and efficacy.

Third, and relatedly, is the dimension of public health ethics, which addresses the question of making new non-validated interventions widely available prior to testing with clinical trials. This question is particularly relevant during emergencies, when infrastructure for randomized controlled trials may not be in place for diseases with a high mortality rate (see [14]).

In this article, we evaluate a highly popularized NNVP for AD management, metabolic enhancement protocols, and argue that there are ethical issues with its current use and promotion along the three ethical dimensions identified—clinical ethics, scientific communication ethics, and public health ethics. Recognizing that the ethical requirements mentioned in paragraph 37 of 
the Helsinki declaration seem insufficient for guiding responsible NNVP in the treatment of AD, we propose a provisional set of principles and benchmarks to address this gap in the literature.

\section{Metabolic enhancement protocols as innovative practice}

Recent observational studies reveal several modifiable risk factors for dementia: low education, physical inactivity, obesity, midlife smoking, depression, hypertension, and diabetes. It is thought that improving such risk factors could prevent or delay $40 \%$ of dementia cases $[6,15]$. The US National Institute on Aging nevertheless recognizes that "the quality of evidence falls short" of supporting a full-blown public health campaign to promote such interventions [16]. Thus, any practice manipulating biological or lifestyle risk factors for AD is new and nonvalidated according to our definition above.

The most highly popularized innovative intervention for $\mathrm{AD}$ is the metabolic enhancement for neurodegeneration (MEND) protocol, a "personalized therapeutic program" that "involves multiple modalities" [17]: diet, supplementation, sleep, stress, and metabolic markers (e.g., heavy metals, oxidative stress, insulin, inflammation). MEND is based on papers published in 2014 and 2016 by Dale Bredesen and colleagues in the journal Aging [17, 18]. A subsequent study first-authored by Bredesen, published in 2018 in the Journal of Alzheimer's Disease and Parkinsonism, has apparently shown "documented improvement in cognition" for 100 patients following the reversal of cognitive decline protocol (ReCODE) [19], which "uses a specific set of 140+ factors, labs and cognitive and genetic testing" [20]. We collectively bracket MEND/ReCODE under the rubric of metabolic enhancement protocols for AD. 


\section{Clinical ethics: Can metabolic enhancement protocols be used responsibly to treat individual patients?}

Paragraph 37 of Helsinki indicates that physicians may use non-validated interventions with individual patients only "after seeking expert advice" and "with informed consent" [8]. In 2011, Bredesen and colleagues "proposed the first comprehensive, double-blind, placebo-controlled trial for early Alzheimer's disease therapeutics," which, according to Bredesen, was rejected by both private and public institutional review boards (IRBs) for "being too complicated" (quoted in [21]). Thus, the expert advice sought seemed to suggest that his protocol was not suitable to test a meaningful therapeutic hypothesis with $\mathrm{AD}$ patients.

The journal Aging, which published Bredesen's protocol [17, 18], claims to adhere to guidelines elaborated by the Committee on Publication Ethics (COPE) and the recommendations of the International Committee of Medical Journal Editors (ICMJE) [22]. Additionally, the journal welcomes editorials, research papers, theory articles, research perspectives, reviews, and mega-reviews and books, stipulating:

All research involving humans and animals must have been approved by the authors' institutional review board or equivalent committee and that board named by the authors. In the case of human participants, informed consent must have been obtained and all clinical investigation must have been conducted according to the principles expressed in the Declaration of Helsinki. Authors should submit a statement from the ethics committee or institutional review board indicating their approval of the research. [23]

Yet it seems that in Bredesen's case, Aging has published a research paper without mention of its IRB rejection or any statement about consent. For contrast, one might refer to the "ethical and regulatory considerations" offered in Eliane Gluckman and colleagues' landmark paper on an innovative practice case study involving the transplantation of umbilical-cord blood to induce 
hematopoietic reconstitution in an anemic patient [24]. In publishing metabolic enhancement protocols for $\mathrm{AD}$, the authors and platform are equally guilty of neglecting guidelines regarding interventions with human subjects.

Given both the apparent lack of precursory expert support for metabolic enhancement protocols in the form of IRB approval and the absence of any explicit demonstration of informed consent, these new non-validated interventions cannot, under the Declaration of Helsinki, be responsibly used to treat individual $\mathrm{AD}$ patients in clinical settings.

\section{Communication ethics: Have authors and platforms responsibly reported the results of metabolic enhancement protocols?}

In the lay press, Bredesen's 2017 published book The End of Alzheimer's: The First Program to Prevent and Reverse Cognitive Decline [25] is a New York Times and Wall Street Journal bestseller, with over 1500 positive reviews on Amazon.com. Within it, Bredesen claims that "progression to severe dementia has until now been inevitable, with nothing but bad news from every expert. The anti-Alzheimer's protocol my colleagues and I developed consigns that bleak dogma to the dustbins of history" [25, p. 11]. He claims to provide "scientific evidence" that supports his conclusions [25, p. 13]. The headline of a CBN News interview with Dr. Bredesen professes that "new research proves Alzheimer's symptoms can be reversed naturally" [26]. Bredesen is now Chief Science Officer of brain health at Apollo Health LLC, which "provides the only Bredesen-approved protocol for preventing, treating, and reversing Alzheimer's disease and cognitive decline" [27]. Their description credits him with showing the "first successful reversals of cognitive decline in Alzheimer's disease, published in 2014, 2016, and 2018" [28]. 
Within the scientific press, Bredesen et al. (2016) claim in their abstract that the magnitude of cognitive improvement using MEND is "unprecedented, providing additional objective evidence that this programmatic approach to cognitive decline is highly effective," and maintain that such results "have far-reaching implications for the treatment of Alzheimer's disease" [18]. Bredesen et al. (2018) echo the claim of "unprecedented improvements in cognition" using ReCODE [19].

We would expect to see robust, generalizable data supporting these strong claims; this is not so for the "scientific evidence" Bredesen quotes in his book. Given the risk of spreading misinformation, communication of results from NNVP is highly delicate. We argue that authors and platforms have overstated evidence in favor of metabolic enhancement protocols for AD.

For context, these protocols are based in functional medicine, whose proponents claim to address "root cause, rather than symptoms" with treatment that "targets the specific manifestations of disease in each individual" [29]. Unfortunately, functional medicine requires ordering several expensive, unvalidated diagnostic tests and measuring serum levels of dozens of micronutrients and cofactors, as well as prescribing "corrective" supplements. The American Academy of Family Physicians found that "a lack of accompanying evidence existed to support the practice of Functional Medicine" and observed some treatments to be "harmful and dangerous" (quoted in [30]). Proponents of functional medicine dismiss such criticism, contending that research models are unable "to test each individualized, patient-centered therapeutic plan that is tailored to a person with a unique combination of existing conditions, genetic influences, environmental exposures, and lifestyle choices" [31]. Bredesen et al. (2018) in turn criticize failed "monotherapeutic" pharmaceutical strategies for AD for "targeting the mediators (e.g., A $\beta$ peptides) instead of the root causes (e.g., pathogens, toxins, and insulin 
resistance)," defending their "very different approach" that involves "addressing the many potential contributors to cognitive decline for each patient"- the apparent success of which "implies that the root cause(s) of the degenerative process are being targeted, and thus the process itself is impacted" [19].

However, the "each patient" individualism touted in the studies by Bredesen and colleagues $[17,18,19]$ undermines their ability to demonstrate improvements. To begin with, all evaluations in medicine require a basis for comparison: a treatment is better or worse than another or no treatment. Since the authors do not have a non-treatment (control) group and also use a radically individualized methodology, the only comparisons possible are between the same patients before and after their study. Yet the three papers provide only sparse descriptions of patients' cognitive decline. They do not convey how radical the changes due to the protocol were in patients' lives; all they allege is that the protocol helped them improve. Given the difficulty of establishing improvement using before-and-after longitudinal evaluations, contemporary medical method uses cross-sectional comparisons between treatment and non-treatment control groups in randomized controlled trials (RCTs) in order to infer causal claims about hopeful treatments and overcome uncertainty about their safety and efficacy [32].

Another problem is the lack of published selection criteria: the authors do not explain why certain patients' data were published and not others, and so a bias to publish data from patients who improved cannot be ruled out. The absence of controls also means that placebo/enrollment effects may be partly responsible for the improvements. Finally, there is no evidence that the studies were blinded so as to avoid bias when collecting or interpreting data. For these reasons, we do not agree that the authors have demonstrated the protocols to halt cognitive decline. 
However, supposing patients did improve, making generalized claims requires some measurement consistency, which all three studies appear to lack. In Bredesen's 2014 study, only three out of ten patients' improvements from MEND are described in detail: patient 1 "noted that all of her symptoms had abated"; for patient 2, "his wife, co-workers, and he all noted improvement"; patient 3 "no longer needed her iPad for notes, and no longer needed to record conversations" [17]. While clinically relevant, such improvements are vague. The "status" of the rest of the patients is summarized primarily using one to three words: normal, improved, or working (except patient 9 who also had "negative amyloid PET" and patient 10 whose status is “decline").

In their 2016 paper, Bredesen et al. describe ten patients' improvements from MEND and identify their apolipoprotein E (ApoE) genotype, a genetic risk factor for Alzheimer's [18]. However, in tabulating their treatment outcomes, each participant is described in terms of “subjective improvement," "marked subjective improvement," or (in one case) "clear subjective improvement," without any explicit operational distinction drawn between the descriptors (e.g., “marked" vs. "clear"). Moreover, the objective treatment outcomes listed are inconsistent and at times vague: patient 1 had "hippocampal volume increase"; patients 6 and 7 had improved minimental state exam (MMSE) scores; patient 9 had improved Montreal cognitive assessment (MoCA) scores; and patients 2-5, 8, and 10 all had general "neuropsychological testing improvement."

Similar issues surface in Bredesen and colleagues' 2018 presentation of 100 patients' results with ReCODE [19]. While only three cases are described in detail, the authors ostensibly tabulate participants' ApoE genotype, symptoms, diagnosis, evaluation, follow-up, and comments. Yet a closer look reveals that their table has numerous blanks-ApoE genotyping is 
not performed for twenty patients, symptoms are omitted for patient 50, twenty-eight patients have no reported evaluation, and patients 87 and 93 have no reported follow-up. Moreover, different evaluation and follow-up methods are used across individual patients without justification: for example, why are qEEG and MoCA used with patient 27, MRI and SLUMS used with patient 44, and FDG-PET and MMSE used with patient $76 ?^{1}$ Additionally, the table shows unexplained discrepancies between objective follow-up results and subjective assessments. In particular, patient 61 has a very low MoCA score which is seen to decrease ("MoCA $5 \rightarrow$ Declined"), and yet the comment describes him as "vastly improved, conversing again, dressing himself, calling grandchildren by name, working again." That a patient with such a low MoCA score could be "working again" seems improbable without further explanation. It does not follow from individualized treatment, potentially justified by AD's complexity, that there is good reason for individualized evaluation of cognitive outcomes.

Ultimately, these functional-medicine-style individualized measures mean that patient improvements as a result of metabolic enhancement protocols cannot be generalized.

Descriptions of subjective outcomes are opaque, and the reasons for use of different objective measurements are not made explicit. Furthermore, reliance on "subjective improvement" may skew the results insofar as decreasing awareness of cognitive decline is recognized as a marker of worsening early AD [33]. Finally, none of the papers contains statistical significance tests or a methods section, making the findings more difficult to reproduce and thereby undermining the principle that NNVP should be validated through research [7].

\footnotetext{
${ }^{1}$ For reference, the methods are respectively abbreviated as follows: quantitative electroencephalogram (qEEG), Montreal cognitive assessment (MoCA), magnetic resonance imaging (MRI), Saint Louis University Mental Status (SLUMS), fluorodeoxyglucose-positron emission tomography (FDG-PET), and mini-mental state examination (MMSE).
} 
We therefore consider the claims made by Bredesen et al. $[17,18,19]$ to be overstatement, given the paucity of supporting data consisting in case studies without selection/exclusion criteria, controls, blinding, consistent metrics, or significance tests. These statements violate paragraph 36 of Helsinki [8]. A further ethical dimension of the overstatement is its intention, which we cannot fully establish here [34].

Additionally, Bredesen and colleagues' 2018 paper was published in the Journal of Alzheimer's Disease and Parkinsonism [19], which is part of OMICS Publishing Group. OMICS has been recognized by Jeffrey Beall as a predatory Open-Access publisher [35], with PubMed Central blacklisting many OMICS publications [36] —indeed, the 2018 paper by Bredesen et al. is not indexed on PubMed. Regarding the other two papers [17, 18], Aging was listed by Beall as possibly predatory [37].

For an example of more balanced language with innovative interventions, take Tiia Ngandu and colleagues' 2015 Finnish geriatric intervention study to prevent cognitive impairment and disability (FINGER), in which 591 at-risk elderly individuals underwent a two-year multidomain intervention to prevent cognitive decline compared to a 599-person control group [38]. Finding statistically significant differences in cognition between these groups, the authors claim in their abstract that "findings from this large, long-term, randomised controlled trial suggest that a multidomain intervention could improve or maintain cognitive functioning in at-risk elderly people from the general population" [38] (our emphasis). This RCT was designed specifically to overcome uncertainty regarding treatment outcomes [32], allowing for synchronic comparisons and using consistent measures. Ngandu et al. used their protocol with 591 patients, in contrast to the 10 patients treated with MEND [18], and employed statistical methods to draw conclusions; and yet their language is still far more restrained than that of Bredesen and colleagues, whose 
2016 claim to have findings of "unprecedented" magnitude is, incidentally, undermined by this study's existence.

Guidelines for more balanced communication already exist. For authors of observational studies, the Strengthening the Reporting of Observational Studies in Epidemiology (STROBE) initiative provides a twenty-two-item checklist concerning the title, abstract, methods, results, and discussion of manuscripts before submission to journals [39]. We call on Aging and the Journal of Alzheimer's Disease and Parkinsonism as scientific platforms to respect the guidelines laid out by COPE and the recommendations of the ICMJE, both of which (as mentioned above) Aging already purports to adhere to [22]. For lay platforms, the Code of Ethics for the Society of Professional Journalists offers four principles: Seek truth and report it, minimize harm, act independently, and be accountable and transparent [40]. In table 1, we offer an example of an alternative to overstatements made by Bredesen [25] and one of his platforms in lay press (CBN News) [26].

Table 1. Improving overstatements for innovation in $\mathrm{AD}$ treatment.

\begin{tabular}{|c|c|}
\hline Overstatement & Suggested non-overstatement \\
\hline \multicolumn{2}{|l|}{ Source: Bredesen 2017 [25] } \\
\hline $\begin{array}{l}\text { Let me say this as clearly as I can: } \\
\text { Alzheimer's disease can be prevented, and in } \\
\text { many cases its associated cognitive decline } \\
\text { can be reversed. For that is precisely what my } \\
\text { colleagues and I have shown in peer-reviewed } \\
\text { studies in leading medical journals-studies } \\
\text { that, for the first time, describe exactly this } \\
\text { remarkable result in patients. }\end{array}$ & $\begin{array}{l}\text { Let me say this as clearly as I can: } \\
\text { Alzheimer's disease could eventually be } \\
\text { prevented, and in many cases its associated } \\
\text { cognitive decline could be improved. I believe } \\
\text { this because my colleagues and I have } \\
\text { reported instances of cognitive improvement } \\
\text { in patients adhering to a metabolic } \\
\text { enhancement protocol in published } \\
\text { observational studies. }\end{array}$ \\
\hline Source: CBN News 2019 [26] & \\
\hline
\end{tabular}


New research proves Alzheimer's symptoms can be reversed naturally
Observational studies suggest complex protocol might improve Alzheimer's symptoms

\section{Public health ethics: Should metabolic enhancement protocols be made widely available based on current evidence?}

We cannot support widespread use of metabolic enhancement protocols in the regular health care of $\mathrm{AD}$ patients for reasons that are evidential, financial, and informational.

To start, useful treatments are "intervention ensembles" — that is, not only materials (e.g., a drug), but also information (e.g., dose, scheduling, secondary effects) about the practices that make them safe and efficacious [41]. Given the number of variables involved in these protocols, confecting a useful intervention ensemble — via diet, fasting, rigorous exercise, dozens of supplements - for each AD patient is a colossal undertaking. Bredesen recognizes its complexity, calling it a "silver buckshot" approach [21]. These exacting lifestyle changes could be justified by their potential to deliver therapeutic fruits, but we argue that the current evidence base does not support the likelihood of such outcomes for this treatment-resistant population.

Bredesen nevertheless claims in chapter 1 of his book that "if enough people adopt ReCODE, the consequences would ripple across the nation and the world, cutting medical costs by many billions of dollars a year, preventing Medicare's bankruptcy, reducing the global burden of dementia, and enhancing longevity. All of these are feasible" [25, p. 15]. While we recognize the importance of general lifestyle interventions for overall health and improvement of disease prognosis, these specific claims are unfounded. There are an estimated 5.8 million people over 65 living with AD in the United States in 2020 [42], and Bredesen speculates that there are 
around 75 million Americans at risk of AD by virtue of having the $\varepsilon 4$ version of the ApoE gene [25, p. 100], a gene involved in cholesterol metabolism. The basic cost of procuring an initial one-time ReCODE report runs between $\$ 1,090$ (at a laboratory facility) and $\$ 1,245$ (for a mobile blood draw) [20]. For every at-risk patient to receive such a report would therefore cost approximately \$82-\$93 billion, around 13-14\% of the \$644 billion Medicare budget in 2019 [43, p. 19]. The cost of dietary supplements alone "may range from $\$ 150$ to $\$ 450$ per month" [20]. At a $\$ 300$ average, that would cost $\$ 22.5$ billion per month, over $40 \%$ of the monthly 2019 Medicare budget. Furthermore, a preliminary look at the online customer reviews of Bredesen's book suggests the possibility for greater individual financial harm, with a one-star review reading: "We paid 30k [to] be part of the official immersion training program and have spent thousands more implementing the protocol. We have not had success and none of the other patients we are in touch with have either" [44].

Even correcting the above figures to take into account economy of a scalable protocol, these measures still have a serious potential for financial harm at the individual and federal levels, while also exploiting the hope of patients and their families with low probability of success. We consider multidomain AD interventions using RCT methodology, such as those in the WorldWide FINGERS network [45]—whose US Study to Protect Brain Health Through Lifestyle Intervention to Reduce Risk (US POINTER) is led by the Alzheimer's Association [46], the largest non-profit organization funding $\mathrm{AD}$ research — to present a superior alternative.

Furthermore, we argue that it is unreasonable to expect patients or legal representatives to evaluate the data supporting claims made by a neurologist, so metabolic enhancement protocols cannot currently be used with informed consent insofar as overstatements by their authors have given rise to misinformation. The way that terms like "innovation" are deployed has been shown 
to affect participants' understanding of experimental treatments and thereby their ability to offer informed consent [47].

Due to the potential for financial harm and false hope, granting individual patients responsible access to the interventions advanced by Bredesen and colleagues would require extensive communication of these protocols' evidential limitations to the different stakeholders. Eligible patients should also be made aware of alternative programs, specifically actively recruiting RCTs, so that widespread NNVP does not crowd out more generalizable research [10, 11, 14]. Notably, the ReCODE clinical trial registered in ClinicalTrials.gov in March 2019, which is slated for completion in December 2020 (NCT03883633), is another case-only observational study [48]. In its current state, we would consider widespread adoption of the protocol by clinicians an unfortunate instance of what Jake Earl calls "runaway diffusion" [9], leading to the frustration of generalizable knowledge based on RCTs [14].

\section{Conclusion and guidelines for responsible innovation in treating AD}

Generalizable therapeutic interventions are desperately needed for AD, for which no diseasemodifying treatment currently exists. Solutions may well come from multidomain interventions addressing the multifactorial nature of AD; however, individualized metabolic enhancement protocols, an innovative practice we examined, do not have robust evidence to support their use in general health care. Nevertheless, despite the protocols' having ostensibly been rejected for RCT testing by IRBs, their authors have published data from observational studies and made overstatements about their efficacy in the scientific and lay press, which has led to interest towards the approach from lay platforms and non-experts [49]. We call upon authors and 
platforms (both scientific and lay press) to adhere to ethical codes regulating communication within their field.

Furthermore, there are more generalizable alternatives: multidomain FINGER prevention studies are recruiting, and many hopeful pharmacological treatments are in the AD pipeline. Further promotion of metabolic enhancement protocols risks deceiving hopeful stakeholders, causing financial harm, undermining informed consent, and crowding out sound research pathways.

We conclude by proposing more stringent criteria for innovation than those laid out in the Declaration of Helsinki, which attempt to address the risks that we have identified in this article (table 2). Our proposal is inspired by a narrow selection of ethical works on the use of innovative practice [7], unproven interventions outside clinical trials [50], and first-in-human use [51]. As a disclaimer, our proposed modifications are a limited and incomplete attempt to capture important criteria in an alternative way. In this way, they are meant neither to replace paragraph 37 of the Declaration of Helsinki nor to be used in real-world ethical evaluation of innovative practice. For this, the relevant authorities and medical associations should develop a complete set of ethical guidelines for responsible innovative practice. It is our hope that the criteria we advance here will add impetus to such development, while encouraging critical examination of new nonvalidated practice in the treatment of Alzheimer's disease and other diseases that presently lack disease-modifying therapies. 
Table 2. Proposed adaptations to paragraph 37 of the Declaration of Helsinki regarding "use of unproven interventions in clinical practice" for responsible innovative practice for Alzheimer's disease treatment

\begin{tabular}{|c|c|}
\hline Original & $\begin{array}{l}\text { In the treatment of an individual patient, where proven interventions do not } \\
\text { exist or other known interventions have been ineffective, the physician, after } \\
\text { seeking expert advice, with informed consent from the patient or a legally } \\
\text { authorised representative, may use an unproven intervention if in the } \\
\text { physician's judgement it offers hope of saving life, re-establishing health or } \\
\text { alleviating suffering. This intervention should subsequently be made the object } \\
\text { of research, designed to evaluate its safety and efficacy. In all cases, new } \\
\text { information must be recorded and, where appropriate, made publicly available. }\end{array}$ \\
\hline $\begin{array}{l}\text { Proposed } \\
\text { modifications }\end{array}$ & $\begin{array}{l}\text { 1. Priority of research over innovative practice } \\
\text { In the treatment of an individual patient with Alzheimer's disease, where } \\
\text { proven interventions do not exist or other known interventions have been } \\
\text { ineffective, the physician, recognizing the need for generalizable solutions for } \\
\text { this large treatment-resistant patient population, should first (i) suggest } \\
\text { enrollment in actively recruiting randomized controlled trials (RCTs) or (ii) } \\
\text { seek institutional review board (IRB) approval for an RCT of a hopeful but } \\
\text { non-validated treatment, adhering to classical research pathways and } \\
\text { guidelines. } \\
\text { 2. Independent regulatory and ethical oversight } \\
\text { However, if an IRB rejects RCT testing of the non-validated treatment, and it } \\
\text { is impossible or infeasible to enroll the patient in other RCTs (e.g., no ongoing } \\
\text { eligible trials, prohibitive distance to trial site), then relevant national } \\
\text { regulatory agencies (or other appropriate health authorities) and IRBs (or other } \\
\text { appropriate ethics committees without conflicts of interest and with the } \\
\text { capability to evaluate scientific evidence and perform a benefit-harm } \\
\text { assessment of the intervention) may nevertheless approve the treatment's use } \\
\text { in a monitored protocol of practice with unproven interventions. This use } \\
\text { should not foreseeably hinder or interfere with the initiation, conduct, or } \\
\text { completion, of present or future clinical AD research. } \\
\text { The evaluative capacities of appropriate authorities and committees should be } \\
\text { proportional to the degree of uncertainty or risk and previous experience with } \\
\text { the use of the proposed intervention: higher uncertainty and risk, and less } \\
\text { experience with an unproven intervention, will require that reviewers have } \\
\text { greater and more nuanced capacities for evaluating pre-clinical and scientific } \\
\text { data. } \\
\text { 3. Risk minimization } \\
\text { This activity should be performed in an institutional context with appropriate } \\
\text { resources to ensure that risks can be minimized. } \\
\text { 4. Consent process }\end{array}$ \\
\hline
\end{tabular}


With informed consent from the patient and a legally authorized representative, or with only the legally authorized representative's consent where national regulations allow it, the physician may use an unproven intervention if in the physician's judgement - if and only if not contradicted by an IRB or appropriate independent committee - it offers hope of saving life, reestablishing health or alleviating suffering.

The informed consent form should communicate at least the intervention's and protocol's evidential limitations and main therapeutic intention, the existence of validated interventions or available recruiting RCTs as alternatives, risks and potential benefits, the patient's right to discontinue or refuse the intervention, anticipation of new evidence that may require the intervention to be paused or stopped, and whether patient insurance coverage is unaffected or the patient knowingly chooses self-payment, as well as additional consent for use of data for future research, measures to protect patient privacy, and any other relevant information needed for a valid consent process according to the IRB or appropriate ethics committee. The consent process should be continuous, and patients and representatives should be informed of any change in the evidence that significantly affects the relative risk and potential benefit profile of the intervention.

\section{Registry, data gathering, transparency, and good publishing practices}

Data from monitored protocols of practice with unproven interventions, along with IRB or other appropriate independent committee decisions (e.g., rejection of research but approval of practice), should be registered, documented, and subsequently published as observational research, respecting guidelines for observational studies (such as the STROBE initiative) to minimize the risk of overstatement in the scientific press. In all cases, new information must be recorded, and an accountable third party should approve lay press publication.

National research registries or other appropriate registries should allow for registration of monitored protocols of practice with unproven interventions, and such protocols should clearly be distinguished from generalizable AD research protocols.

\section{Transition to research}

Finally, if the observational data appear promising (e.g., if the treatment outcomes are statistically significant), a new IRB review can be sought in order to undertake generalizable research designed to evaluate the treatment's safety and efficacy.

Acknowledgments The authors thank Katelyn MacDougald for her invaluable copyediting. Timothy Daly thanks the Fondation Médéric Alzheimer for financial support for his doctoral work, and acknowledges helpful support and input from Hervé Maisonneuve, Anouk 
Barberousse, Mathieu Bourdenx, and Felicitas Holzer. 


\section{References}

1. Beauchamp, Tom L., and Yashar Saghai. 2012. The historical foundations of the researchpractice distinction in bioethics. Theoretical Medicine and Bioethics 33: 45-56.

2. National Commission for the Protection of Human Subjects of Biomedical and Behavioral Research. 1979. The Belmont report: Ethical principles and guidelines for the protection of human subjects of research. Washington, DC: US Government Printing Office.

3. Davis, Dena S. 2017. Ethical issues in Alzheimer's disease research involving human subjects. Journal of Medical Ethics 43: 852-856.

4. Jack, Clifford R., Jr., David A. Bennett, Kaj Blennow, Maria C. Carrillo, Billy Dunn, Samantha Budd Haeberlein, David M. Holtzman, et al. 2018. NIA-AA research framework: Toward a biological definition of Alzheimer's disease. Alzheimer's and Dementia 14: 535562.

5. Selkoe Dennis J., and John Hardy. 2016. The amyloid hypothesis of Alzheimer's disease at 25 years. EMBO Molecular Medicine 8: 595-608.

6. Livingston, Gill, Andrew Sommerlad, Vasiliki Orgeta, Sergi G. Costafreda, Jonathan Huntley, Davis Ames, Clive Ballard, et al. 2017. Dementia prevention, intervention, and care. Lancet 390: 2673-2734.

7. Taylor, Patrick L. 2010. Overseeing innovative therapy without mistaking it for research: A function-based model based on old truths, new capacities, and lessons from stem cells. Journal of Law, Medicine and Ethics 38: 286-302.

8. World Medical Association. 2013. World Medical Association Declaration of Helsinki: Ethical principles for medical research involving human subjects. JAMA 310: 2191-2194.

9. Earl, Jake. 2019. Innovative practice, clinical research, and the ethical advancement of medicine. American Journal of Bioethics 19(6): 7-18.

10. Agich, George J. 2019. Knowing one's way around: the challenge of identifying and overseeing innovations in patient care. American Journal of Bioethics 19(6): 1-3.

11. Levine, Robert J. 1979. Clarifying the concepts of research ethics. Hastings Center Report 9(3): 21-26.

12. Mastroleo, Ignacio, and Felicitas Holzer. 2019. New non-validated practice: An enhanced definition of innovative practice for medicine. Law, Innovation and Technology 12: 318346.

13. Haber, Noah, Emily R. Smith, Ellen Moscoe, Kathryn Andrews, Robin Audy, Winnie Bell, Alana T. Brennan, et al. 2018. Causal language and strength of inference in academic and media articles shared in social media (CLAIMS): A systematic review. PLOS ONE 13(5): e0196346. https://doi.org/10.1371/journal.pone.0196346.

14. London, Alex John. 2019. Social value, clinical equipoise, and research in a public health emergency. Bioethics 33: 326-334. 
15. Livingston, Gill, Jonathan Huntley, Andrew Sommerlad, David Ames, Clive Ballard, Sube Banerjee, and Carol Brayne. 2020. Dementia prevention, intervention, and care: 2020 report of the Lancet Commission. Lancet 396: 413-446.

16. Sohn, Emily. 2018. A quest to stave off the inevitable: The idea that certain lifestyle choices could help to prevent Alzheimer's disease is gaining broader acceptance. Nature 559: S18S20.

17. Bredesen, Dale E. 2014. Reversal of cognitive decline: a novel therapeutic program. Aging 6: 707-717.

18. Bredesen, Dale E., Edwin C. Amos, Jonathan Canick, Mary Ackerley, Cyrus Raji, Milan Fiala, and Jamila Ahdidan. 2016. Reversal of cognitive decline in Alzheimer's disease. Aging 8: 1250-1258.

19. Bredesen, Dale E., Kenneth Sharlin, David Jenkins, Miki Okuno, Wes Youngberg, Sharon Hausman Cohen, Anne Stefani, et al. 2018. Reversal of cognitive decline: 100 patients. Journal of Alzheimer's Disease and Parkinsonism 8(5): 1000450. http://doi.org/10.4172/2161-0460.1000450.

20. Apollo Health. 2020. Frequently asked questions. https://web.archive.org/web/20200716220221/https:/www.apollohealthco.com/frequentlyasked-questions. Accessed July 16, 2020.

21. Gustafson, Craig. 2015. Dale E. Bredesen, MD: Reversing cognitive decline. Integrative Medicine 14(5): 26-29.

22. Impact Journals. 2020. Publication ethics and publication malpractice statements. Aging. https://www.aging-us.com/ethics. Accessed March 2, 2020.

23. Impact Journals. 2020. Information for authors. Aging. https://www.aging-us.com/forauthors. Accessed March 2, 2020.

24. Gluckman, Eliane, Hal E. Broxmeyer, Arleen D. Auerbach, Henry S. Friedman, Gordon W. Douglas, Agnès Devergie, Hélène Esperou, et al. 1989. Hematopoietic reconstitution in a patient with Fanconi's anemia by means of umbilical-cord blood from an HLA-identical sibling. New England Journal of Medicine 321: 1174-1178.

25. Bredesen, Dale E. 2017. The end of Alzheimer's: The first program to prevent and reverse cognitive decline. New York: Avery.

26. CBN News. New research proves Alzheimer's symptoms can be reversed naturally. $C B N$ News, April 24, 2019. https://www1.cbn.com/cbnnews/health/2019/april/new-researchproves-alzheimers-symptoms-can-be-reversed-naturally.

27. Apollo Health. 2020. Hope through science. https://web.archive.org/web/20200525224055/https://www.apollohealthco.com. Accessed May 25, 2020.

28. Apollo Health. 2020. Leadership. https://web.archive.org/web/20200525193926/https://www.apollohealthco.com/leadership. Accessed May 25, 2020. 
29. Institute for Functional Medicine. 2020. Functional Medicine determines how and why illness occurs and restores health by addressing the root causes of disease for each individual. https://web.archive.org/web/20200421095351/https://www.ifm.org/functionalmedicine. Accessed April 21, 2020.

30. Mack, Amy. AAFP Announces Decision on Functional Medicine CME. The Institute for Functional Medicine. https://www.ifm.org/news-insights/news-aafp-announces-decisionfunctional-medicine-cme. Accessed January 2020.

31. Functional Medicine South Africa. "The Science." https://www.functionalmedicinesa.co.za/copy-of-functional-integrative-medi. Accessed: $12 / 2019$.

32. Djulbegovic, Benjamin. 2001. Acknowledgment of uncertainty: A fundamental means to ensure scientific and ethical validity in clinical research. Current Oncology Reports 3(5): 389-395.

33. Cacciamani, Federica, Caroline Tandetnik, Geoffroy Gagliardi, Hugo Bertin, Marie-Odile Habert, Harald Hampel, Laurie Boukadida, Marie Révillon, Stephane Epelbaum, and Bruno Dubois. 2017. Low cognitive awareness, but not complaint, is a good marker of preclinical Alzheimer's disease. Journal of Alzheimer's Disease 59: 753-762.

34. Dresser, Rebecca. 1993. Defining scientific misconduct: The relevance of mental state. JAMA 269: 895-897.

35. Beall, Jeffrey. 2010. Update: Predatory Open-Access scholarly publishers. Charleston Advisor 12: 50. https://doi.org/10.5260/chara.12.1.50.

36. Kaiser, Jocelyn. 2013. U.S. government accuses Open Access publisher of trademark infringement. Science, May 9, 2013. https://www.sciencemag.org/news/2013/05/usgovernment-accuses-open-access-publisher-trademark-infringement.

37. Beall, Jeffrey. 2017. List of standalone journals. Scholarly Open Access. Updated January 9, 2017. https://web.archive.org/web/20170111172309/https://scholarlyoa.com/individualjournals.

38. Ngandu, Tiia, Jenni Lehtisalo, Alina Solomon, Esko Levälahti, Satu Ahtiluoto, Riitta Antikainen, Lars Bäckman, Tuomo Hänninen, et al. 2015. A 2 year multidomain intervention of diet, exercise, cognitive training, and vascular risk monitoring versus control to prevent cognitive decline in at-risk elderly people (FINGER): A randomised controlled Trial. Lancet 385: 2255-2263.

39. Vandenbroucke, Jan P., Erik von Elm, Douglas G. Altman, Peter C. Gøtzsche, Cynthia D. Mulrow, Stuart J. Pocock, Charles Poole, James J. Schlesselman, and Matthias Egger. 2014. Strengthening the reporting of observational studies in epidemiology (STROBE): Explanation and elaboration. International Journal of Surgery 12: 1500-1524.

40. Society of Professional Journalists. 2014. Code of ethics. Revised September 6, 2014. https://www.spj.org/ethicscode.asp.

41. Kimmelman, Jonathan, and Alex John London. 2015. The structure of clinical translation: Efficiency, information, and ethics. Hastings Center Report 45(2): 27-39. 
42. Hebert, Liesi E., Jennifer Weuve, Paul A. Scherr, and Denis A. Evans. 2013. Alzheimer disease in the United States (2010-2050) estimated using the 2010 census. Neurology 80: 1778-1783.

43. Congressional Budget Office. 2020. The budget and economic outlook: 2020 to 2030. Washington, DC: Congressional Budget Office. https:/www.cbo.gov/system/files/202001/56020-CBO-Outlook.pdf.

44. C.K. 2018. Beware! Review of The end of Alzheimer's: The first program to prevent and reverse cognitive decline, by Dale E. Bredesen. Amazon.com, August 28, 2018. https://www.amazon.com/gp/customerreviews/R1YWBSWB9HIUN2/ref=cm_cr_getr_d_rvw_ttl?ie=UTF8\&ASIN=0735216207.

45. Rosenberg, A., F. Mangialasche, T. Ngandu, A. Solomon, and M. Kivipelto. 2020. Multidomain interventions to prevent cognitive impairment, Alzheimer's disease, and dementia: From FINGER to World-Wide FINGERS. Journal of Prevention of Alzheimer's Disease 7: 29-36.

46. Baker, Laura D., Daniel P. Beavers, MaryJo Cleveland, Claire E. Day, Charles Decarli, Mark A. Espeland, Sarah E. Tomaszewski-Farias, et al. 2019. O4- 11- 03: U.S. Pointer: Study design and launch. Alzheimer's and Dementia 15(7S): P1262-P1263. https://doi.org/10.1016/j.jalz.2019.06.4802.

47. Richards, Bernadette, and Katrina Hutchison. 2016. Consent to innovative treatment: No need for a new legal test. Journal of Law and Medicine 23: 938-948.

48. QuesGen Systems. 2020. Reversal of Cognitive Decline (ReCODE) Study (RECODE) (Identifier NCT03883633). Updated March 18, 2020. https://clinicaltrials.gov/ct2/show/NCT03883633.

49. Hellmuth, Joanna. 2020. Can we trust The End of Alzheimer's? Lancet Neurology 19: 389390.

50. Pan American Health Organization. 2020. Reliance for emergency use authorization of medicines and other health technologies in a pandemic (e.g. COVID-19). https://covid19evidence.paho.org/handle/20.500.12663/1189

51. Kimmelman, Jonathan, and Carole Federico. 2017. Consider drug efficacy before first-inhuman trials. Nature 542: 25-27. https://doi.org/10.1038/542025a. 